\title{
Study on Reconstruction Accuracy using shapiness index of morphological transformations
}

\author{
P.Radhakrishnan \\ Department of Computer Science \\ College of Computer Science, King Khalid University, Abha, P.O.Box: 394 \\ Kingdom of Saudi Arabia, Phone: 00966-7-2417019 \\ http://radhakrishnan.info \\ Email: radha.krish5@gmail.com, krish_res@yahoo.com
}

\begin{abstract}
Basin, lakes, and pore-grain space are important geophysical shapes, which can fit with the several classical and fractal binary shapes, are processed by employing morphological transformations, and methods. The decomposition of skeleton network (minimum morphological information) using various classical structures like square, octagon and rhombus. Then derive the dilated subsets respective degree by the structures for reconstruct the original image. Through shapiness index of pattern spectrum procedure, we try test the reconstruction accuracy in a quantitative manner. It gives some general procedure to characterise the shape-size complexity of surface water body. The reconstruction accuracy is against the size of water bodies with which we produce the some example of different shapiness index for different structuring element of shapes. In which quantitative manner approach yields better reconstruction level. The complexity of water bodies are compared with the surfaces.
\end{abstract}

\section{Keywords}

Reconstruction, Skeleton network, Mathematical morphology, Pattern spectrum, Shape-size complexity.

\section{Introduction}

Geophysical process may understand through Shape description. The geophysical basins, elevation regions, lakes, rock pore-grain space are closely identified through the simplified shapes. This investigation includes shapes for importance from the point of geophysical studies. Using certain indicators of geophysical science can be determined from the overall shape. The channel network or pore-grain connectivity network can be treated as minimum information employing which one can make an attempt to reproduce the original shapes. For example the basin processes can be used for the spatial organization of the channel network patterns. This minimum information has highly significant, morphological information, where we can rebuild the basin. Using morphological transformations and rules we can identify minimum information. To check the correctness of the constructing basin by analysing with original information in spatial domain, pattern spectrum procedure is of immense use. These study considering various binary synthetic images, which is from threshold of elevation regions (DEM) [Sagar, 2000]. The connectivity network patterns converted from binary shapes, which have minimum morphological information in the basin, i. e., channel network patterns. To reconstruct the original shapes we use these connectivity network subsets. We will analysis the reconstructing importance through various predefined morphological rules and pattern spectrum procedures. Several studies have been carried out of which the procedure based on mathematical morphology, though DOI : $10.5121 /$ ijcseit.2012.2601 
computationally expensive, proved to be robust. So in a way there is a link between minimum morphological information and reconstruction rules. This study emphasized to explore the potentiality of several existing procedures, which are essentially based on morphological transformations. Present investigation deals with a treatise on the applications of morphological transformations to investigate shapes from geophysical aspect. Various procedures are adapted to achieve an aim include superficially simple but elegant mathematical morphology transformations such as erosion, dilation, opening and closing. These transformations are systematically used associated with different logical operations. In whole of the study, the reader realizes the importance of the rules, which we term as structuring element.

In this paper geostatistical tools have been employed to quantify the degree of accuracy in the reconstructed basins, and to compute shapiness indices of several geophysical phenomena. Several synthetic shapes and connectivity networks, which are termed as geophysical basins and networks, are considered in this chapter. The organisation of the connectivity network pattern determines the basin processes. In order to verify the accuracy in such a reconstructed basin by comparing with original basin's organisation in spatial domain, pattern spectrum procedure is adopted. However, in this chapter, various synthetic basins in binary form, which are akin to thresholded elevation regions of DEM [Sagar, 2000] and surface water bodies are considered. These synthetic elevation regions are converted into possible connectivity networks, which are akin to the minimum morphological information in the basin, (e.g. channel network pattern). The subsets of connectivity network are the main focus from which the original synthetic thresholded elevation regions are constructed. Generation of connectivity networks, their reduced versions, and reconstruction of basins from these two types of networks are targeted in this chapter. The terms such as image, shape which are often used are the shapes of geophysical interest, structuring element term is the probing rule with which the shapes of geophysical interest will be reconstructed, and the terms "connectivity network or the skeleton network are akin to the unique networks such as channel, flow direction and pore-grain connectivity networks.

\section{Morphological Transformations}

Mathematical morphology [P.Maragos, 1987, P.Radhakrishnan 2005] is a set algebra used to process and analyze data based on geometric shapes. Structuring element which is small pattern is used to examine the geometrical structure of an image. The $X$ is defined as finite Euclidean twodimensional space $Z^{2}$. Let $B$ denote a structuring element, which is a subset in $Z^{2}$ with a simple geometrical shape and certain characteristic information. The base image and structuring element are used for morphological operations. This operation will process the images and produce the resultant image by specifying the size of structuring element. Figure 1shows the structuring template of different shape like octagon, square, and rhombus which are used for reconstruction accuracy for any image. The four basic morphological transformations are erosion, dilation, cascade of erosion-dilation and dilation-erosion. The mathematical representation of these four fundamental morphological transformations is given in equation (1), the diagrammatic representation can be seen from the literature.

$$
\begin{array}{ll}
\text { Erosion: } & X \Theta(-B)=\{x: B+x \subset X\} \\
\text { Dilation: } & X \oplus(-B)=\{x: B+x \cap \subset X \neq \varnothing\} \\
\text { Opening: } & X \circ B=[X \Theta(-B)] \oplus B \\
\text { Closing: } & X \bullet B=[X \oplus(-B)] \Theta B
\end{array}
$$


The opening and closing nonlinear transformations can be implemented iteratively by following multi-scale approaches, in which the size of the SE will be incremented from iteration to iteration. In the multi-scale approach, the size of the B will be increased form iteration to iteration [P.A.Maragos 1987].

$$
n B=\underset{n \text { times }}{B \oplus B \oplus B \oplus \ldots \oplus B}
$$

where $n$ is the discrete size parameter. Using nonlinear transformations, we can compute the shapes on various aspects. To characterize the normal shapes we apply the reconstruction procedure and pattern spectrum procedure. Utilize the standard four fundamental transformations which can decompose a binary shape, akin to geophysical shape, and a skeleton to reconstruct the shape from the skeleton. Also these application are also applied in pattern spectrum procedure for verify the standard of reconstructions. One pixel size channel we can derive by applying the various structuring element in morphology. These thinner lines are of special interest because they explain the structure of the original basins and vertices. The distance of skeleton is the line made up of those points to the boundary which includes the beginning and end of thinner line. Highly symmetrical objects have the skeletons with symmetry. The more irregular is the object, the more irregular is its skeleton. Morphologically the skeleton extraction phase can be achieved by connectivity the basic morphological transformation as shown in equation $(3,4)$

$$
\begin{aligned}
& S k(X)=(X \Theta n B) \backslash\{[(X \Theta n B) \Theta B] \oplus B\} \quad n=0,1,2, \ldots, N \\
& S k(X)=\bigcup_{n=0}^{N} S k_{n}(X)
\end{aligned}
$$

where $S k_{n}(X)$ denotes the $n t h$ skeletal subset of shape $(X)$. Morphological erosion is used for $\mathrm{X}$ and structuring element for $\mathrm{n}$ times which will be followed by opening by $\mathrm{B}$, finally subtracting from the eroded versions of $X$ their opening by $B$. The morphological skeletal network will be produced from the union of all such possible points. The given shape will be decomposed as skeletal network by using reconstruction with same structuring element which is used in computation of skeleton.

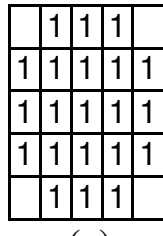

(a)

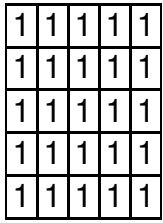

(b)

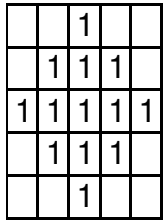

(c)

Figure 1. Binary patterns in $\mathrm{Z}^{2}$ (SE), (a) octagon, (b) square, and (c) rhombus

\subsection{Reconstruction of binary image}

Reconstruction is one classical way in mathematical morphology to achieve robustness by conditioning transformations to a reference set or image, and therefore controlling the spatial extensions of the morphological transformations. Morphological reconstruction is a process of taking a partial connected component and recreating the entire connected component, based on the intensities in the input image $X$. Successive dilations are preformed on the image with markers, each dilation being followed by an intersection with the original image until 
convergence or idempotence. Reconstruction retrieves the original shape of the retained particles after erosion, which eliminates small objects. Skeleton is precisely decomposed from its shape by the morphological operations that is designed precisely. In the next phase, these skeletal subsets, decomposed [Tun-Wen Pai, 1994] from the shape are dilated by an explicit number of iterations by way of trying to reconstruct the shape outline by means of certain predefined morphological rule. Through pattern spectrum procedure [8], we derive shapeiness index (I) for the binary images. The morphological analog of frequency in conventional signal processing, is size. Hence any geometric spectrum, obtained morphologically [P.A.Maragos 1987], is a measure of sizecontent (of the structuring element) in the given image. Thus such a geometric spectrum of an image can be plotted against the size (of the structuring element) axis. The $n^{\text {th }}$ entry in the pattern spectrum [Eqn.5] is defined as:

$$
\begin{aligned}
& P S(n)=\text { Area }\{X \circ n B \backslash X \circ(n+1) B\}, n=0,1, \ldots, N_{\max -1} \\
& P S(n)=0, n \geq N_{\max }
\end{aligned}
$$

where $X$ is the image and $B$ is the structuring element, $S \backslash Q=\{x \in S: x \notin Q\}, \mathrm{N}_{\max }$ is the minimum size of the $B$, such that the erosion of image $X$ with $N_{\max } B$ results in the null set. Thus the $n^{\text {th }}$ entry in the pattern spectrum is the cardinality of the set difference between the opening of the image $X$ by the structuring element $B$ of size $n$ and $(n+1)$.It is a shape-size descriptor, which can detect critical scales in an image object and quantify various aspects of its shape-size content. Since opening removes the portion smaller than the SE, the difference of the images opened by the SE of size $n$ and size $n+l$ contains the portion whose size is exactly $n$. The number of pixels in the set obtained by subtracting the opened objects from the original one gives the area of those objects that cannot contain the SE. Thus, iterative application of the morphological opening and the measurement of the residues, while increasing the size of the SE gives the size distribution of the objects contained in the given image.

\subsection{Study of reconstruction accuracy}

Mathematical morphological transformations are employed [BSD Sagar,1998] to decompose a binary shape by means of various SE. To study the aspects of binary image the morphological procedures are applied in integrated process, which includes derivation of morphological rules for topological structure. To derive these rules several binary shapes are simulated and the procedure based on mathematical morphology has been systematically implemented to verify the accuracy of morphological rules and in the reconstructed shapes [Eq. 6].

$$
\begin{aligned}
& X=U_{n=1}^{N}\left[S_{n}(X) \oplus n B\right] \\
& \text { where } s_{n}(x)=((X \Theta n B) \backslash(X \Theta n B) \circ B)
\end{aligned}
$$

Pattern spectrum $P S_{x}(r, B)$ is given by (7), where $A(X)$ means that the area of $X$ and $r$ is the scale.

$$
P S_{x}(r, B)=A((X \circ B) \backslash(X \circ r B))
$$

$A(X o r B)$ is a measure of the pattern content of $X$ relative to the pattern $r B$ [P.A.Maragos, 1986]. By varying both $r$ (scale) and the shape of $B$ (structuring element) we obtain a shape-size spectrum of $X$, which is the full pattern spectrum of $X$ relative to all the patterns that can fit inside $X$. The higher a spectrum at the maximum $r$, the more alike an area $X$ is as a structuring element $B$. Shapeiness is a likeness between $X$ and $B . \quad B$-shapeiness $S_{x}(B)$ [Eq. 8] is as follows, where $r_{\max }$ means that maximum of scale $r$. 


$$
\begin{aligned}
& S_{X}(B)=P S\left(r_{\max -1}, B\right) / A(x) \\
& S_{X}(B)=\left(P S_{X}\left(r_{\max }, B\right)+P S_{X}\left(r_{\max -1}, B\right)\right) / A(X)
\end{aligned}
$$

The above calculation yields 1 if the image and structuring element are same. When $r_{\max -1}$ is not maximum, the $S_{X}(B)$ is calculated by applying the $r_{\max -1}$ for pattern spectrum . The procedure is explained in flowchart [Figure 2].

\section{Case Study and Results}

Various planner shapes [Figure. 5] that include both classical and irregular shape have been investigated in discrete space with an aim to estimate the shape-size complexity measure. The different rules applied in discrete space to investigate the shape sixe complexity through the shapiness index. The structuring elements like octagon, square, rhombus and irregular shapes are studied to analysis the reconstruct accuracy [Figure 3]. We plotted all these shapes in one image and carried out the reconstruction procedures. For each shape there is a different pattern spectrum value through different SE like square, octagon, Rhombus [Figures. 4, 6, 7, 8]. These values are given (Table 1-3). Flowchart [Fig. 2] shows various steps involved in the adopted procedure [Figures. 4, 6, 7, 8]. Fractal shape is considered with different SE. It gives 80 to $90 \%$ accuracy of reconstruction. Square shape with square SE gives $100 \%$ accuracy. The reconstruction accuracy varies to $40 \%$ from $10 \%$, which is a lowest level. Similarly the rectangle shape with square SE gives $100 \%$ accuracy but with other SE it gives 10 to $30 \%$ of accuracy.

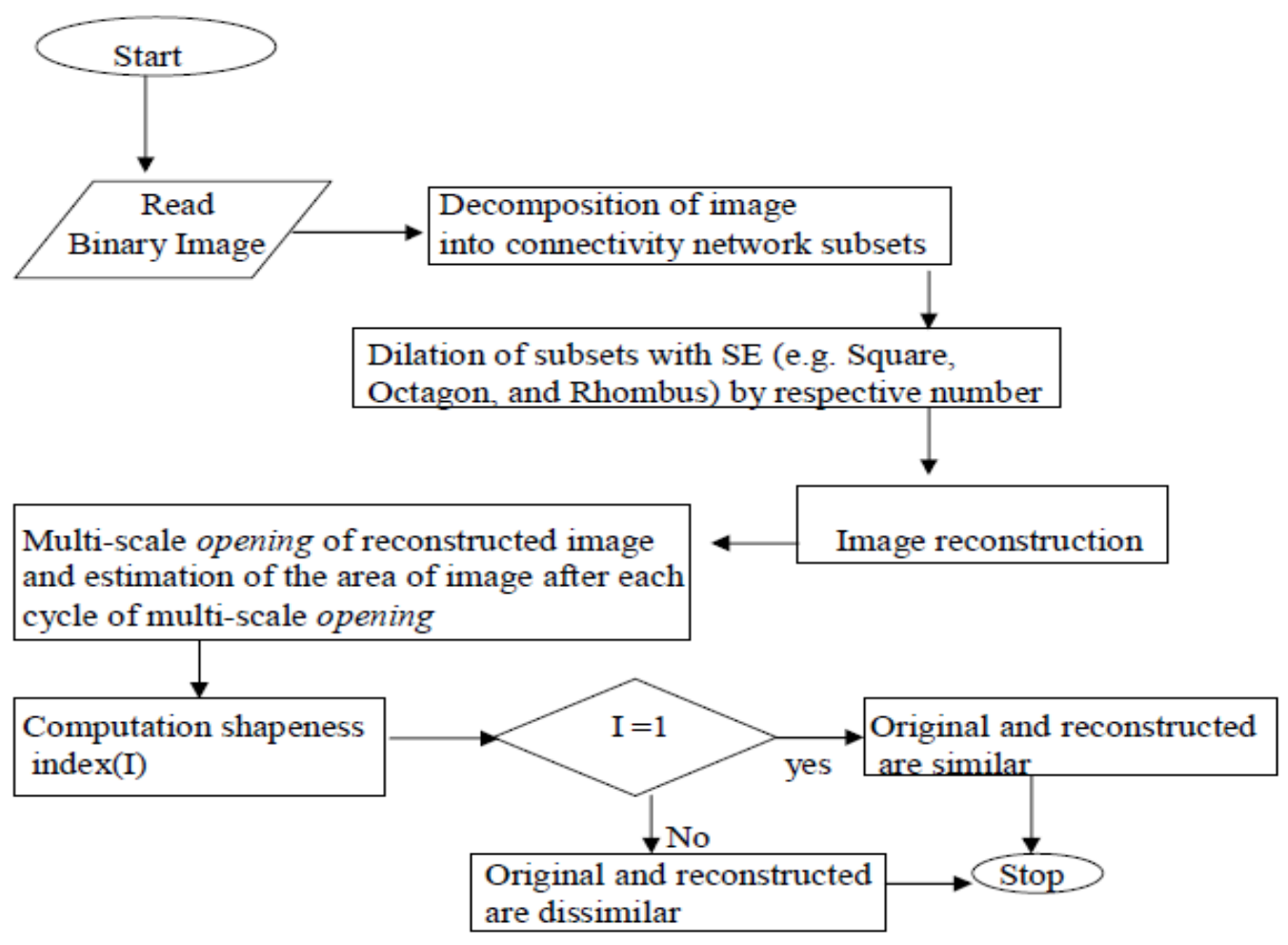

Figure.2 Sequential steps involved in the study of accuracy 
The reconstruction accuracy of the binary shapes have been studied by calculating shapiness index. The range of this index is 0 to 1 . If the shapeiness index is 1 , then the two images are in the same pattern with exact geometric similarity. The dissimilar images yield 0 for shapiness index. Binary image reconstruction includes various recurrences of steps of morphological rules. The first step is to isolate of skeletal subset by means of a specific structuring element (e.g. rhombus). In this step, the skeletal subsets up to $n$th order will be extracted by simply subtracting the opened version of the eroded image from the eroded image. The erosion degree will be varied. This step is better explained through mathematical morphological representation (Eq.4). If each skeletal subset of each degree is dilated to the same degree by the same structuring element, which is used to extract the skeletal subsets, the union of all the dilated skeletal subsets to the specific degree will produce exactly the original binary shape. Obviously, this produced a shape, which is not similar to that of the original shape. This phenomenon is said to be that the reconstruction accuracy is imperfect.
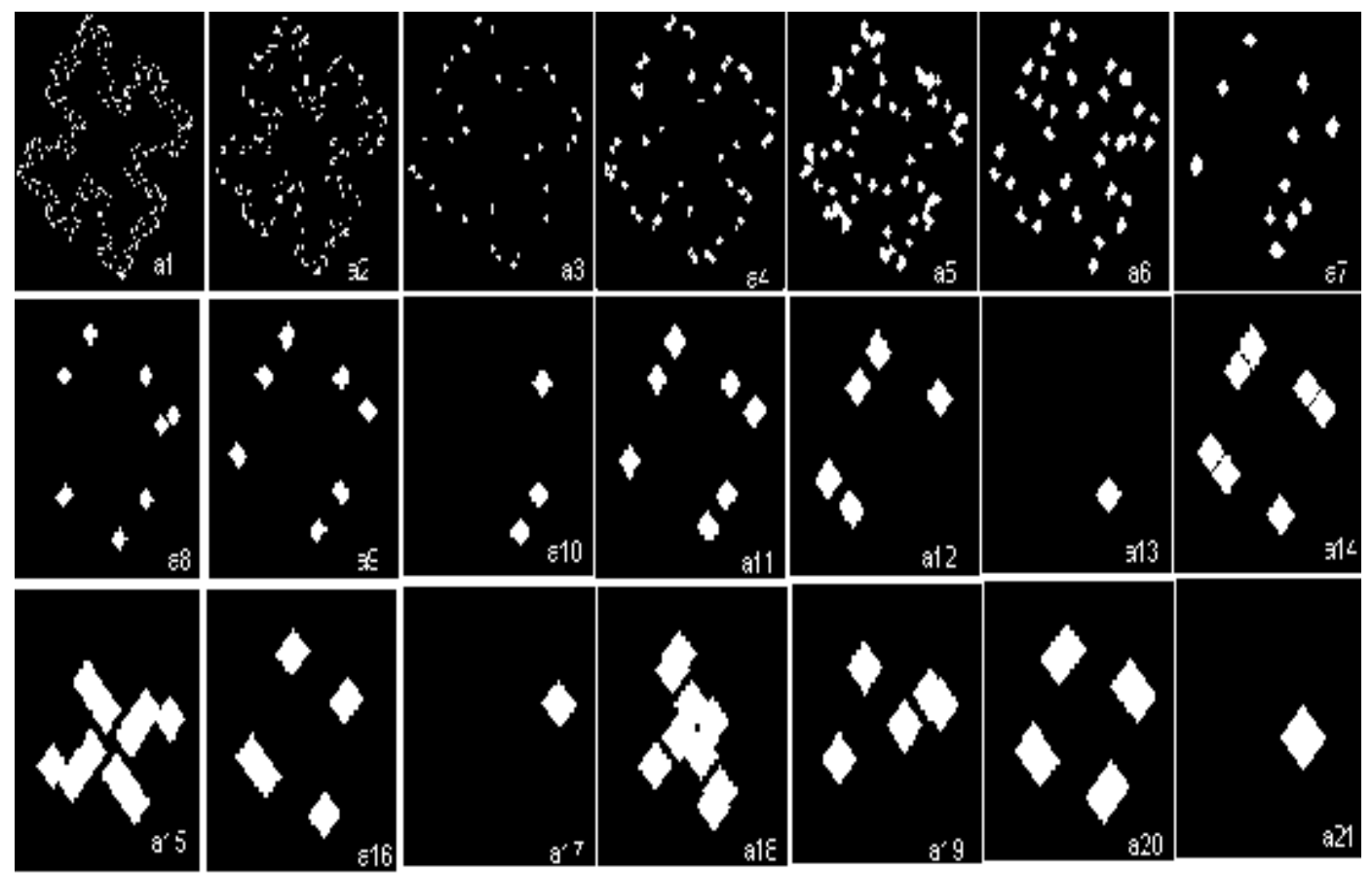

沙 1
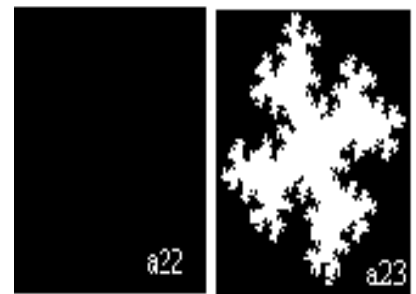

Figure 3 Reconstruction fractal (a simulated TER of DEM) using rhombus as structuring element . a1-a22: different scale subsets of dilation of original image. a23: union of a1-a22. $(a 22=\varnothing)$ 


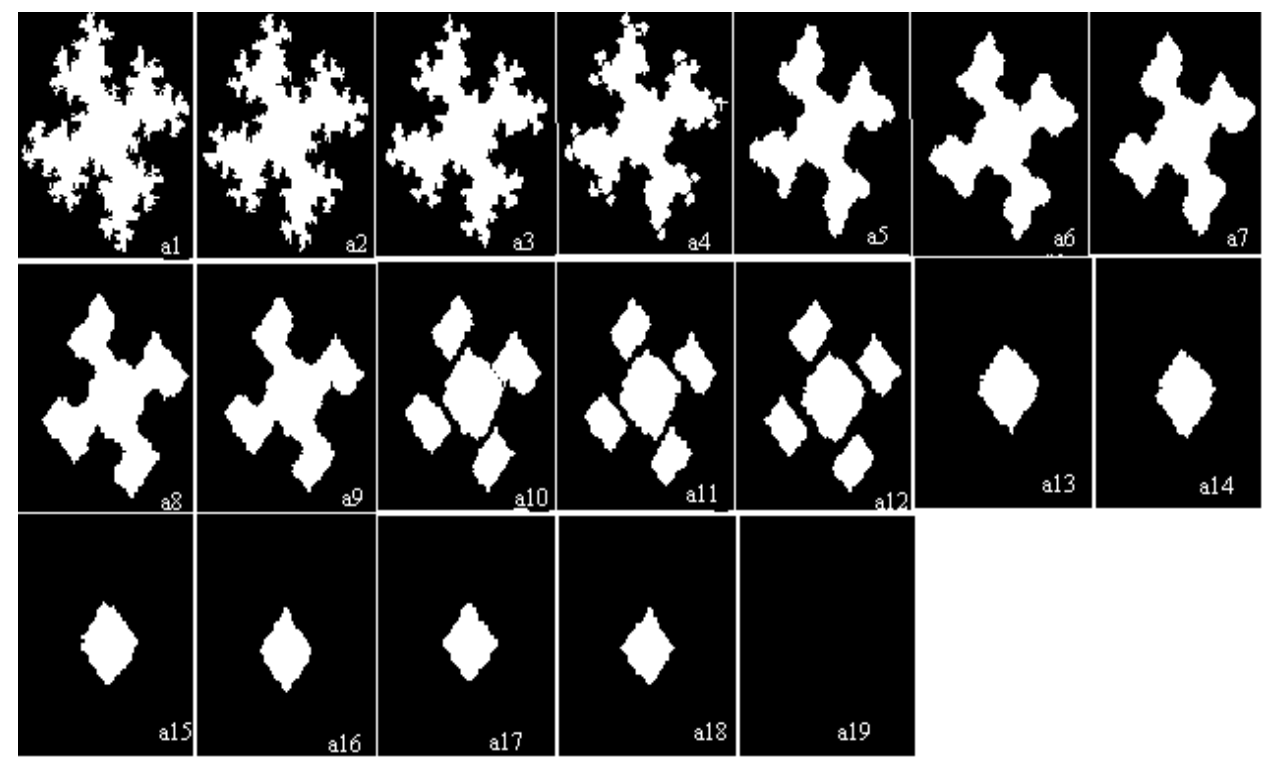

Figure 4 Pattern Spectrum of fractal image with rhombus as structuring element.

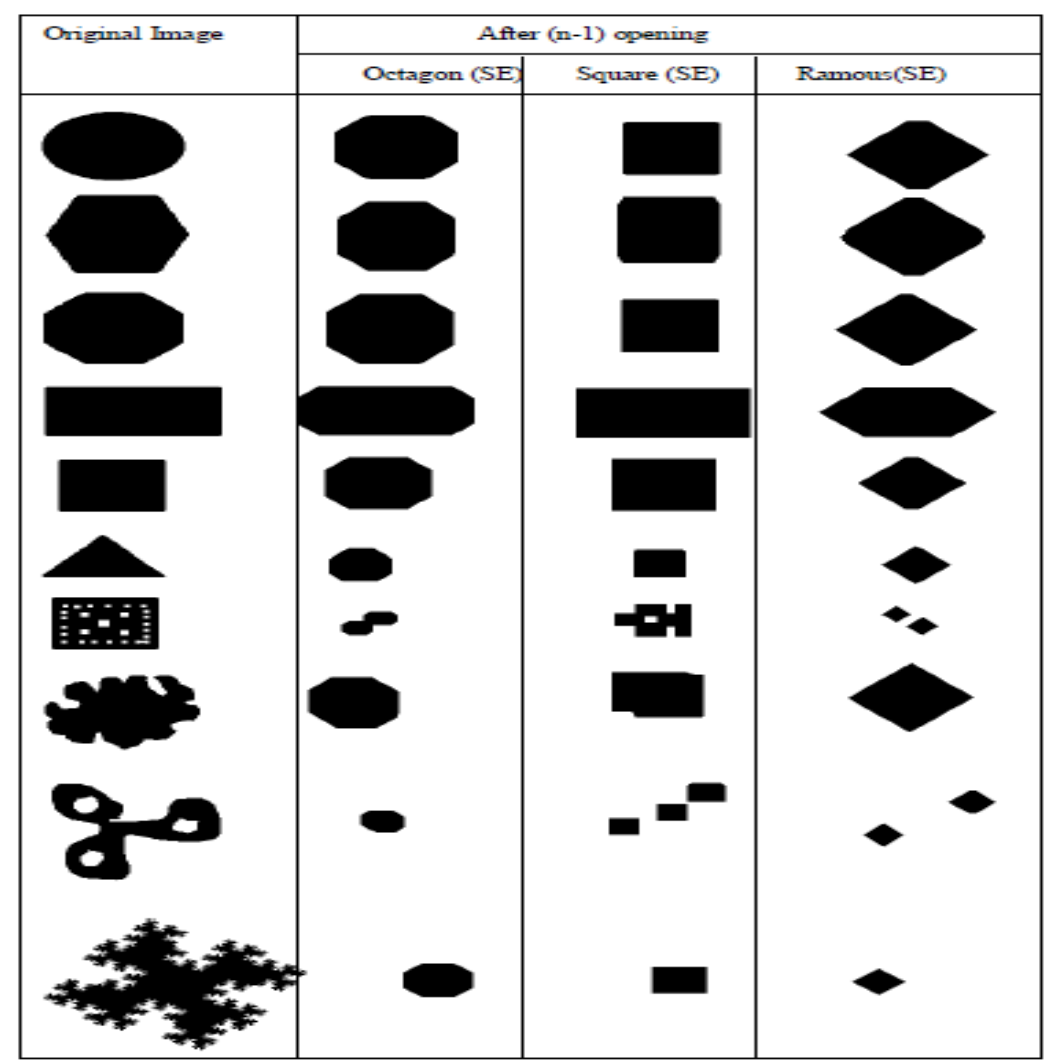

Figure 5. Comparison table of the original basin with n-1 opening of the same basin with different discrete rules. 


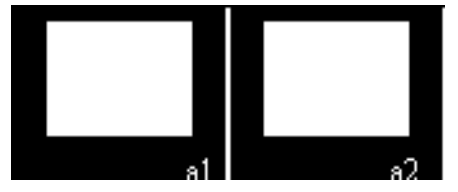

Fig 6 :Pattern spectrum of square with square as structuring element. a1: image with one openings, a2: image with n-1 openings.

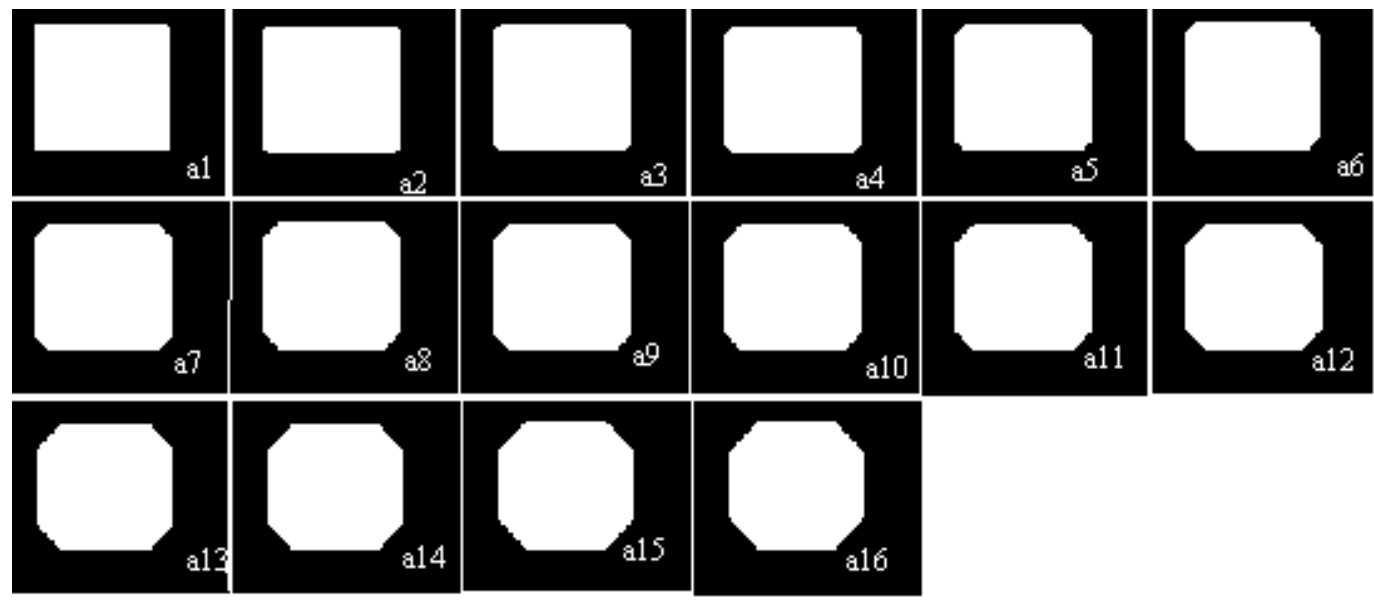

Figure 7 Pattern spectrum of square with octagon as structuring element. a1: image with one openings, a2-a15 different scale openings, a16: image with n-1 openings,

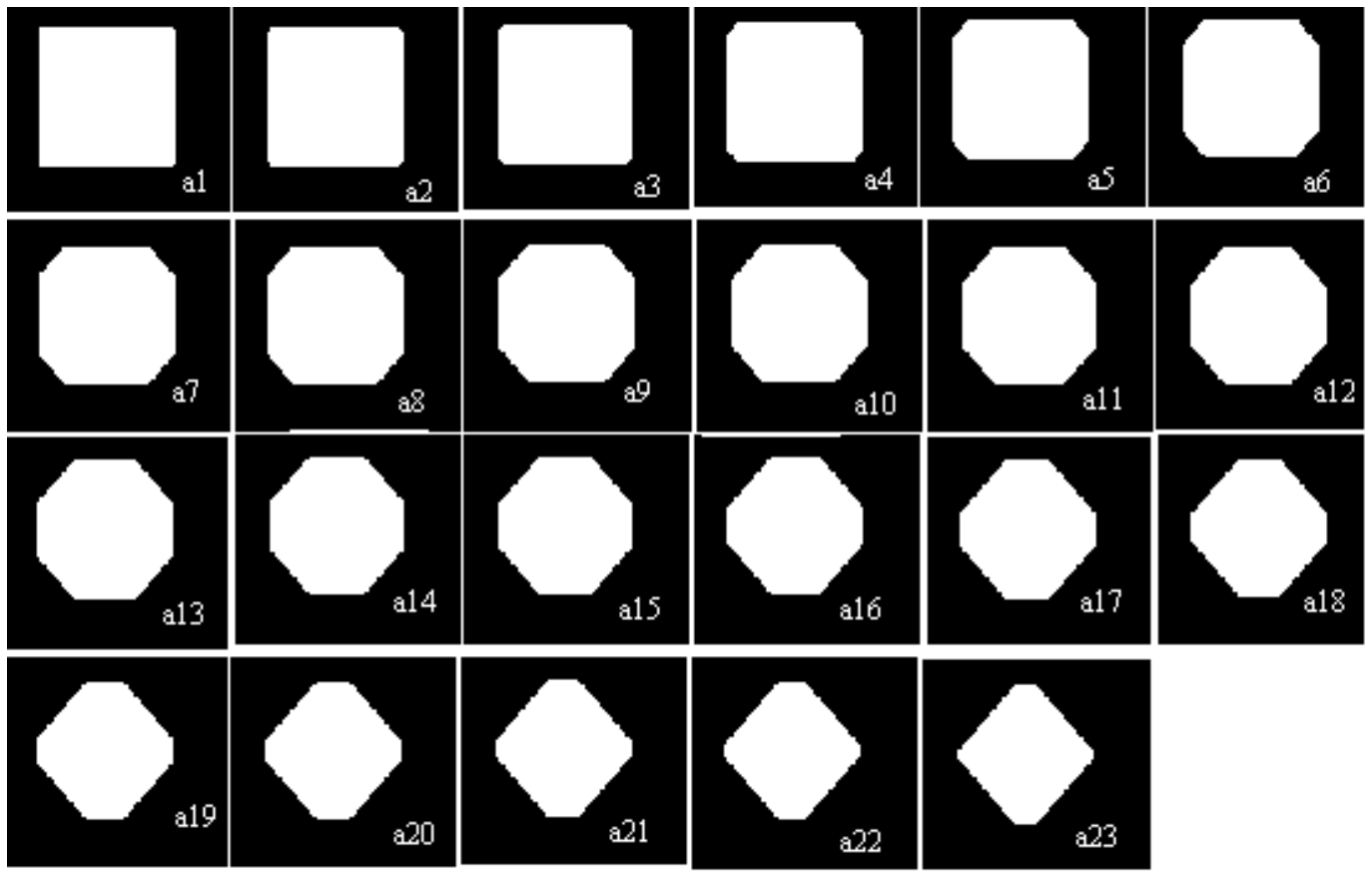

Figure 8: Pattern spectrum of square with rhombus as structuring element. a1: image with one openings, a2-a22 different scale openings, a23: image with n-1 openings. 


\subsection{Small water bodies}

Analysis the shape size complexity we considered the 35 number of small water bodies (Figure 9) situated randomly over a $10 \mathrm{sq}$. km landscape, which produces general trend to derive a shapesize complexity relationships. We computed the shapiness index for all water bodies by implementing multiscale morphological opening (Table 5). It ranges from 0.2 to 0.83 , which shows that the smaller topological water bodies yields higher shapiness index, so reconstruction accuracy is more on small water bodies. But the higher geometrically water bodies may have some low level accuracy through morphological transformations rules. A simple graph between logarithm of areas of water bodies and their shapiness indices (Figure. 10) are plotted. A general trend indicating the relationship between shape and size is observed, which further can be used to make firm shape-size complexity relationship. However, it is worth considering a large number of water bodies, for instance 300 and over, that may provide a single power-law based on fractallength-area-perimeter-volume dimensional analysis.

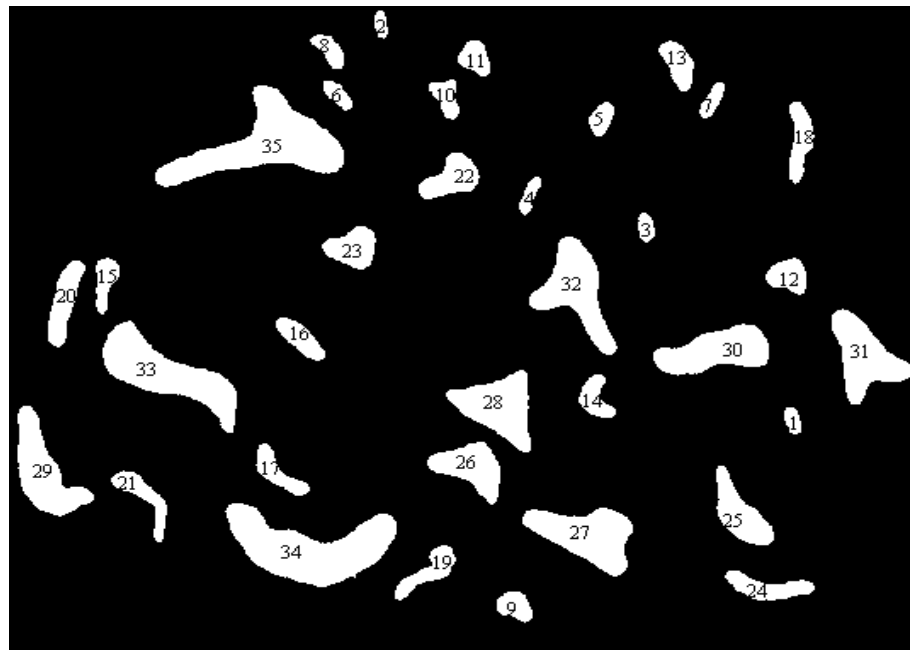

Figure 9: Small water bodies, traced from IRS 1D remotely sensed data, situated in the flood plain region of Gosthani river (A.P) India.

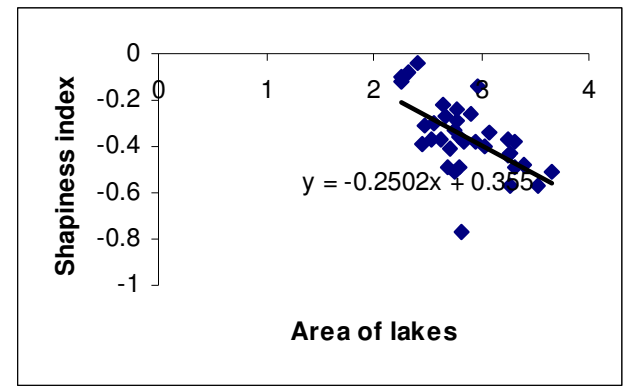

Figure 10: shape-size complexity of small water bodies

In this paper we compare size distributions via the concept of a pattern spectrum. It can be applied through the size distributions of shapiness index in discrete- space binary or gray tone image. From the experimental results, it was shown that the shapeiness index (I) was available information for individual recognition and it could be classified with $100 \%$ accuracy. To improve the classification accuracy, we will consider a new structuring element like ellipse or 
asymmetrical shape. The scale shows the size of structuring element. By statistical bar graph we derive the graph of water bodies and shapiness index of a shape pattern generated [Figure. 11a, $11 b]$ by a prototype pattern.

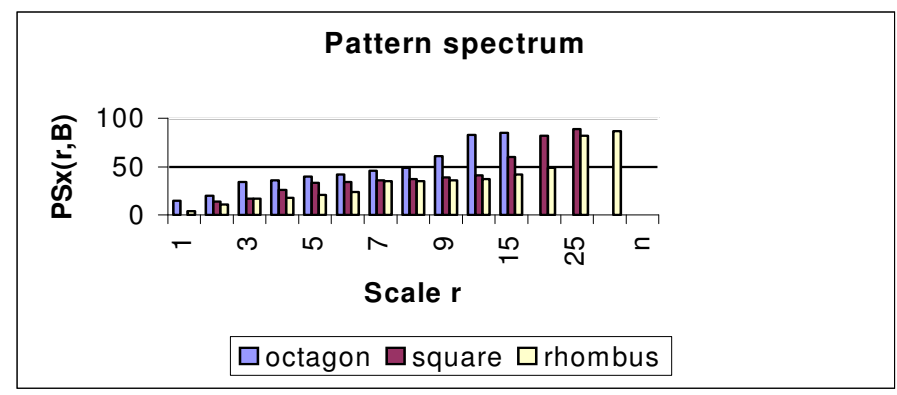

Figure 11a :Pattern spectrum of Fractal

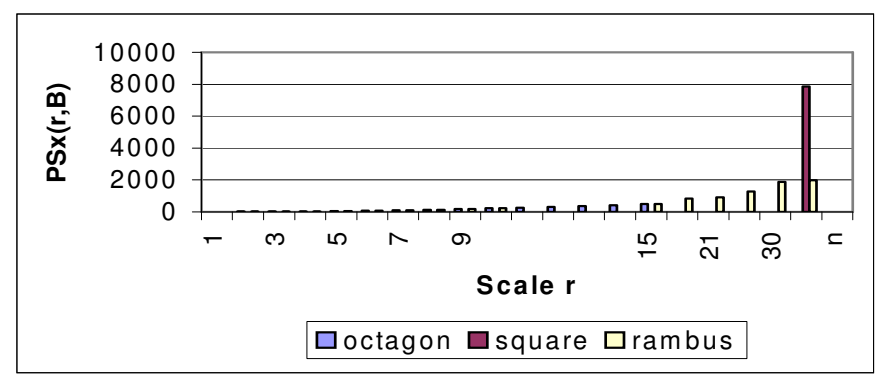

Figure 11b: Pattern Spectrum of Square

Table 1: Pattern spectrum of Fractal

\begin{tabular}{|l|l|l|l|l|l|l|l|l|l|l|l|l|l|l|l|}
\hline Scale & 1 & 2 & 3 & 4 & 5 & 6 & 7 & 8 & 9 & 10 & 15 & 20 & 25 & 38 & n \\
\hline $\mathrm{PS}(16$, Octagon) & 15 & 20 & 34 & 36 & 40 & 42 & 46 & 49 & 61 & 83 & 85 & & & & 0 \\
\hline $\mathrm{PS}(26$, Square $)$ & 0 & 14 & 17 & 26 & 33 & 34 & 36 & 37 & 39 & 41 & 60 & 82 & 89 & & 0 \\
\hline $\mathrm{PS}(39$, Rhombus) & 4 & 11 & 17 & 18 & 21 & 24 & 35 & 35 & 36 & 37 & 42 & 49 & 82 & 87 & 0 \\
\hline
\end{tabular}

Table 2: Pattern spectrum of Square

\begin{tabular}{|l|r|r|r|r|r|r|r|r|r|r|r|r|r|r|r|r|r|}
\hline Scale & 1 & 2 & 3 & 4 & 5 & 6 & 7 & 8 & 9 & 10 & 15 & 16 & 20 & 25 & 30 & 32 & $\mathrm{n}$ \\
\hline PS(17,Octagon) & 4 & 12 & 24 & 40 & 60 & 84 & 112 & 144 & 180 & 220 & 480 & 544 & & & & & 0 \\
\hline PS(33,Square) & 0 & 0 & 0 & 0 & 0 & 0 & 0 & 0 & 0 & 0 & 0 & 0 & 0 & 0 & 0 & 4950 & 0 \\
\hline PS(33,Rhombus) & 4 & 12 & 24 & 40 & 60 & 84 & 112 & 144 & 180 & 220 & 480 & 544 & 840 & 1300 & 1860 & 2112 & 0 \\
\hline
\end{tabular}

Table 3: Shapeiness index I of binary image with different SE.

\begin{tabular}{|l|c|c|c|c|c|c|c|c|}
\hline $\begin{array}{l}\text { Structuring } \\
\text { element IShapes }\end{array}$ & Square & Rectangle & Triangle & Octagon & Hexagon & Circle & $\begin{array}{l}\text { Irregular } \\
\text { Shape }\end{array}$ & Fractal \\
\hline SE(Square) & 1.0 & 1.0 & 0.5 & 0.6 & 0.6 & 0.4 & 0.6 & 0.9 \\
\hline SE(Octagon) & 0.1 & 0.1 & 0.4 & 1.0 & 0.4 & 0.9 & 0.8 & 0.8 \\
\hline SE(Rhombus) & 0.4 & 0.3 & 0.5 & 0.5 & 0.7 & 0.4 & 0.7 & 0.9 \\
\hline
\end{tabular}


Table 4: Pattern spectrum(PS(n)), network transform $\left(S_{n}\right)$, and $\operatorname{RST}\left(R_{n}\right)$ of the fractal basin of Figure.3

\begin{tabular}{|r|c|r|r|r|r|r|r|}
\hline $\mathbf{N}$ & $\mathbf{P S ( n )}$ & \multicolumn{1}{c|}{$\mathbf{S}_{\mathbf{n}}$} & $\mathbf{R}_{\mathbf{n}}$ & $\mathbf{N}$ & $\mathbf{P S ( n )}$ & $\mathbf{S}_{\mathbf{n}}$ & $\mathbf{R}_{\mathbf{n}}$ \\
\hline 0 & 47552 & 47752 & 47552 & 10 & 28159 & 223 & 219 \\
\hline 1 & 47552 & 403 & 403 & 11 & 27005 & 123 & 109 \\
\hline 2 & 47552 & 405 & 405 & 12 & 26406 & 136 & 134 \\
\hline 3 & 47028 & 358 & 342 & 13 & 24972 & 143 & 139 \\
\hline 4 & 46329 & 1066 & 1006 & 14 & 20634 & 122 & 121 \\
\hline 5 & 41668 & 720 & 642 & 15 & 19151 & 125 & 125 \\
\hline 6 & 36225 & 380 & 346 & 16 & 10901 & 68 & 69 \\
\hline 7 & 29225 & 257 & 255 & 17 & 5793 & 47 & 47 \\
\hline 8 & 30696 & 205 & 199 & 18 & 0 & 0 & 0 \\
\hline 9 & 29860 & 248 & 232 & & & & \\
\hline
\end{tabular}

Table 5 Shapiness of indices of randomly situated surface water bodies of various sizes and shapes

\begin{tabular}{|l|r|r|}
\hline Name & \multicolumn{1}{|l|}{ Area } & \multicolumn{1}{|c|}{ Shapiness } \\
\hline Lake1 & 180 & 0.79 \\
\hline Lake2 & 182 & 0.76 \\
\hline Lake3 & 210 & 0.83 \\
\hline Lake4 & 256 & 0.91 \\
\hline Lake5 & 278 & 0.41 \\
\hline Lake6 & 304 & 0.49 \\
\hline Lake7 & 340 & 0.43 \\
\hline Lake8 & 362 & 0.5 \\
\hline Lake9 & 413 & 0.43 \\
\hline lake10 & 443 & 0.61 \\
\hline Lake11 & 454 & 0.53 \\
\hline Lake12 & 497 & 0.33 \\
\hline Lake13 & 512 & 0.39 \\
\hline lake14 & 552 & 0.31 \\
\hline Lake15 & 555 & 0.46 \\
\hline lake16 & 588 & 0.51 \\
\hline Lake17 & 604 & 0.57 \\
\hline
\end{tabular}

\begin{tabular}{|l|r|r|}
\hline Name & \multicolumn{1}{|l|}{ Area } & \multicolumn{1}{l|}{ Shapiness } \\
\hline lake18 & 615 & 0.32 \\
\hline lake19 & 627 & 0.44 \\
\hline lake20 & 668 & 0.17 \\
\hline Lake21 & 697 & 0.41 \\
\hline Lake22 & 818 & 0.55 \\
\hline lake23 & 875 & 0.42 \\
\hline Lake24 & 906 & 0.72 \\
\hline Lake25 & 1051 & 0.4 \\
\hline Lake26 & 1215 & 0.46 \\
\hline Lake27 & 1779 & 0.36 \\
\hline Lake28 & 1792 & 0.42 \\
\hline Lake29 & 1818 & 0.37 \\
\hline Lake30 & 1822 & 0.27 \\
\hline Lake31 & 2010 & 0.32 \\
\hline Lake32 & 2022 & 0.42 \\
\hline Lake33 & 2559 & 0.33 \\
\hline Lake34 & 3326 & 0.27 \\
\hline Lake35 & 4566 & 0.31 \\
\hline
\end{tabular}

\section{Conclusion}

Filling any image by using morphological reconstruction approach yields full-length packing, the efficiency of filling the various shapes including irregular. The higher reconstruction accuracy gives higher ratio of packing efficiency. In quantitative terms, the fractal structural element yields best reconstruction accuracy of shapines index, it can be higher in packing or filling the shape through fractal shapes. The highest reconstruction accuracy value is close to 1 from fractal structural element of the square and rhombus which are 0.9 and 0.8 respectively. In contrast, the shapiness index of the same fractal by means of octagon is 0.8 which can consider as a rule that describes the lower packing efficiency compare to the other two structuring elements. In our 
study, the pattern spectrum procedure is utilized for identifying shapiness index, which starts in normal shapes like square, rhombus, octagon and finally with irregular shapes of water bodies. The water bodies are in different size of geometrically structure which is randomly situated surface water bodies are also considered, and their shapiness indices are computed. The shape size complexity of various water bodies observed with a general trend.

\section{References}

[1] B. S. D. Sagar, M. Venu and D. Srinivas, Morphological operators to extract channel networks from Digital Elevation Models, International Journal of Remote Sensing. 21 (1) (2000) 21-30.

[2] P.Maragos, Pattern spectrum of images and morphological shape-size complexity, Acoustics, Speech, and Signal Processing, IEEE International Conference on ICASSP '87. Apr 1987, Volume: 12, Page(s): 241 - 244

[3] P.Radhakrishnan, Characterization of reconstructed basins using pattern spectrum procedure, American journal of applied sciences vol. 2, issue 4, pp 843 - 846, 2005

[4] P.A. Maragos, Pattern spectrum and multiscale shape representation, IEEE Pattern Analysis and Machine Intelligence 11 (7) (1989).

[5] B.S.D. Sagar, M.Venu, G.Gandhi, and D.Srinivas, Morphological description and interrelation between force and structure: a scope to geomorphic evolution process modeling, Int. J. Remote Sensing 19 (7) (1998) 1341-1358.

[6] Tun-Wen Pai, J.H,L.Hansen, Boundary-Constrained Morphological skeleton minimization and skeleton reconstruction, IEEE Trans on Pattern Analysis and Machine Intelligence 16 (2) (1994).

[7] P.A.Maragos,, R.W. Schafer, Morphological skeleton representation and coding of binary images, IEEE Trans on Acoustics, speech and signal processing ASSP-34 (5) (1986)1228-1244.

\section{Author}

Dr.Radhakrishnan Palanikumar working as Asst. Prof in King Khalid University Saudi Arabia for last 7 years and his teaching experience is more than 17 years in various international universities. His research interest includes image processing and segmentations.

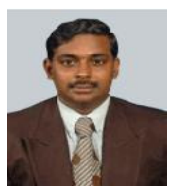

